Title

\title{
Unraveling gene function in agricultural species using gene co-expression networks
}

\author{
Authors and affiliations \\ Robert J. Schaefer ${ }^{1}$, Jean-Michel Michno ${ }^{1}$, Chad L. Myers*1,2 \\ ${ }^{1}$ Biomedical Informatics and Computational Biology Graduate Program, University of Minnesota, \\ Minneapolis, MN 55455 \\ ${ }^{2}$ Department of Computer Science and Engineering, University of Minnesota, Minneapolis, MN 55455 \\ *Corresponding author (cymers@cs.umn.edu)
}

\section{Keywords}

Co-expression, functional genomics, agriculture, crops, network analysis, guilt-by-association

\section{Abstract}

Co-expression networks have been shown to be a powerful tool for inferring a gene's function when little is known about it. With the advent of next generation sequencing technologies, the construction and analysis of co-expression networks is now possible in non-model species, including those with agricultural importance. Here, we review fundamental concepts in the construction and application of co-expression networks with a focus on agricultural crops. We survey past and current applications of co-expression network analysis in several agricultural species and provide perspective on important considerations that arise when analyzing network relationships. We conclude with a perspective on future directions and potential challenges of utilizing this powerful approach in crops.

\section{Introduction}

Despite extremely successful breeding programs substantially impacting agricultural yield, our understanding of gene function in most crop species is still limited. For instance, in maize, only $1 \%$ of genes have functional annotations based on mutant analysis [1]. However, applications of high throughput, genome sequencing technologies are generating data that is beginning to expose the function of the rest of the genome. For example, standardized sequencing-based techniques, such as RNA-Seq, enable the measurement of gene expression for specific experimental conditions, developmental time points or different tissues. Surveying transcription across a large number of diverse experiments establishes an expression profile for each gene, which can be exhaustively compared to each another, building a network of putative co-regulatory relationships. Each node represents a gene and each edge shows the magnitude of co-expression between them, implying a probabilistic, functional relationship [2]. In addition to when and where it is expressed, these data can help establish a functional context for a gene, even where little other information exists.

In model species, where gene function can be established through reverse genetic approaches, coexpression networks have been shown to be a powerful tool for rapidly predicting potential functional links between genes. Furthermore, once represented as a network, topological and structural 
information shows that these biological networks remarkably share organization properties similar to other naturally occurring networks such as those seen in power grids, social interactions, and the world wide web [3]. Borrowing these systems biology based approaches developed in model species, systematic integration of large-scale whole genome expression datasets is now an active area of research in crops. Whole transcriptome sequencing technologies in crop species allows for a transfer of knowledge from decades of previous research in non-biological and model systems to build functional networks using co-expression. This shared domain knowledge allows for the direct application of many network based approaches in crop species allowing for rapid construction of robust, biologically coherent networks. However, there are unique characteristics of agricultural species that require special consideration in the application and interpretation of co-expression network approaches including high levels of nucleotide diversity, prevalent genotype by environment (GxE) interactions, and heterosis [4]. While not all agronomically important traits will be fully explained by variation in gene expression, the wealth of currently available gene expression information already available for many species coupled with the rate at which new expression studies are being performed makes co-expression analysis a powerful tool for unraveling gene function in crop species. The impact of these phenomena on interpreting functional relationships are just beginning to be explored in agricultural species and pose important considerations for applying co-expression based techniques.

In this review, we examine how gene co-expression networks have been used to unravel gene function, with a focus on agricultural species. First, we review generalized methods and techniques used to build and utilize co-expression networks. We then survey different strategies that previously have been used to examine gene function inferred from co-expression in several different crop species. Next, we focus on interpreting and understanding network structure that arises from co-expression networks built specifically in agricultural species. Finally, we provide our perspective on emerging applications of coexpression networks in helping to interpret the vast wealth of functional information that is now being generated for agricultural systems.

\section{Building co-expression networks}

\subsection{Measuring co-expression among pairs of genes}

Early methods in gene expression analysis discovered that when genes were hierarchically clustered, genes with similar biological function generally clustered near each other (Figure 1A). A gene's pattern of expression across different samples is informative of its function [5]. The functional information originally observed in hierarchically clustered gene expression profiles can also be analyzed through the lens of pairwise relationships. Where the basic unit in a gene expression dataset is a gene's expression profile across a diverse set of experiments, the unit of interest in a gene co-expression network arises from quantifying the relationship among two genes' profiles. A typical approach is to systematically compare each pair of genes' profiles, representing similarity of two genes' profile with a single edge in a network, where the nodes represent genes and the edges reflect highly similar profiles (Figure 1B). 


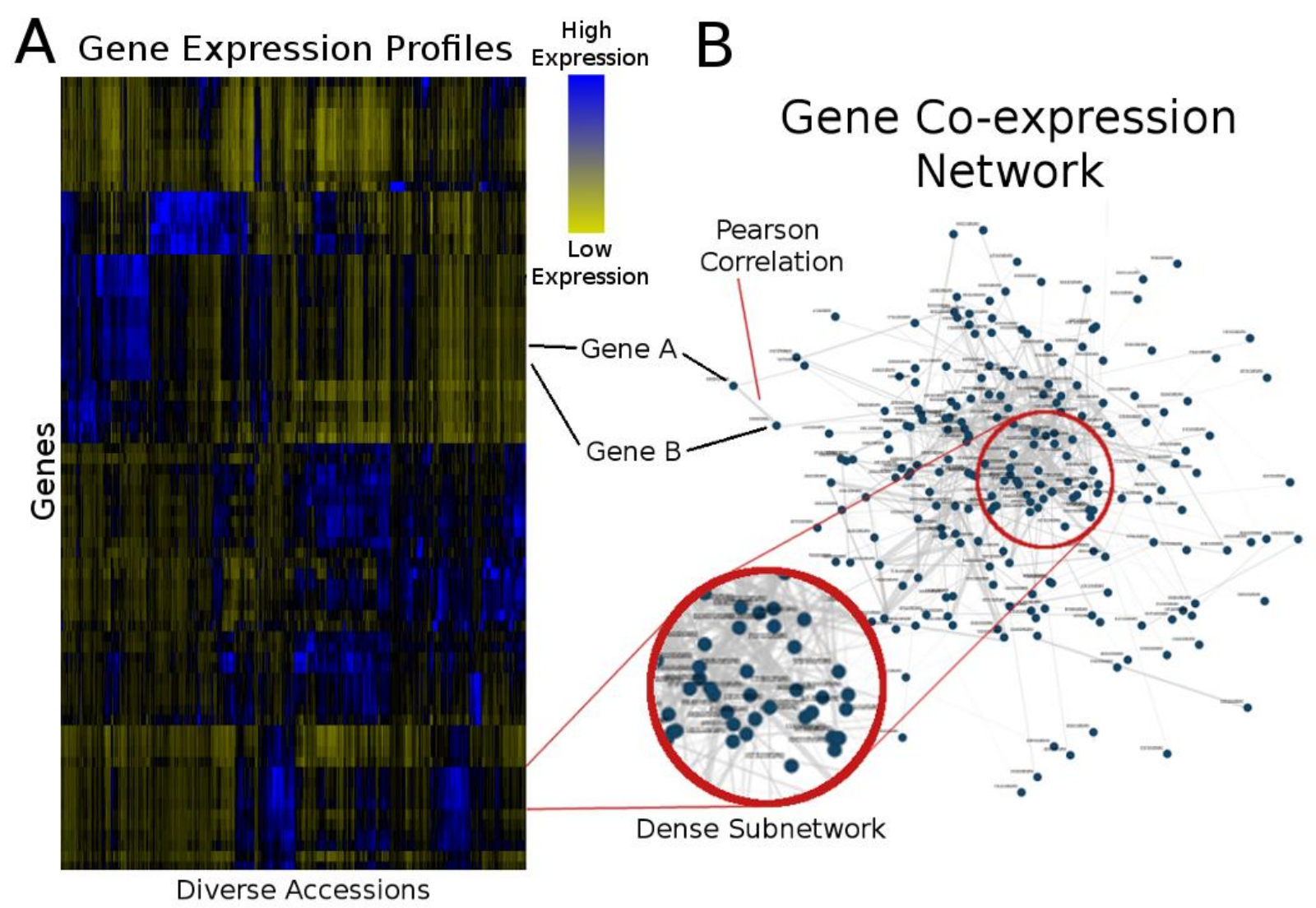

Figure 1

Gene co-expression network construction and overview schematic. (A) Expression is measured for each gene within a genome to generate an expression profile across a diverse set of accessions (often tissues, developmental time-points, stress responses or genotypic variation). Genes are ordered, here, based on a hierarchical clustering algorithm to demonstrate the overall structure captured by the data. (B) Correlative structure can be quantified using a similarity metric (Pearson correlation coefficient here) applied to each pair of genes (Sec. 2.1). Interactions are normalized and thresholded to produce a network and displayed using a 'node' and 'edge' model (Sec 2.2.1). Subnetwork structure, such as dense connectivity, relates back to the original correlation among gene expression profiles captured by variation among the diverse set of accessions. Biological interpretation then relies on understanding why profiles of genes are co-expressed (Sec. 2.2). Patterns here are potentially driven by a subset of accessions being over-or under-expressed, collectively.

The relationship between two genes' expression profiles can be measured several different ways [6]. The most widely used similarity metric used to calculate co-expression is the Pearson correlation coefficient (PCC), which measures the presence of a linear relationship between a pair of expression profiles. This similarity metric has an intuitive interpretation and is computationally inexpensive to compute, which may be factors that contribute to its widespread use for co-expression analyses[2,715]. Despite its extensive use, the Pearson correlation coefficient is not optimal for capturing nonlinear relationships, which possibly leaves meaningful relationships uncovered. Other similarity metrics have been proposed. Non-parametric measures such as the Spearman and Kendall correlation coefficient are alternatives to the PCC and can offer the advantage of robustness to outliers [16-18]. Additionally, more complex measures such as mutual Information (MI) attempt to quantify similarity more generally as a summary of statistical dependence rather than relying on single bi-variate associations, although this comes at the cost of computational efficiency [19]. Recent comparative studies on similarity metrics 
shows that in most cases, linear monotonic relationships capture instances where co-expression is informative suggesting that similarity metrics designed for linear relationships such as PCC are suitable for most general purpose network analysis [20]. Highly similar co-expression patterns among sets of genes are often driven by a small subset of individuals or experiments that are collectively over- or under-expressed (Figure 1A). While determining this subset of accessions is not explicitly handled by common co-expression measures, simultaneous clustering of accession profiles in an approach called biclustering can help determine which accessions are potentially 'driving' patterns of co-expression $[21,22]$. Additional reviews on uses and applications of similarity metrics can be found here $[6,20,23,24]$. For the remainder of this review, we focus on the Pearson correlation coefficient as the basis for measuring co-expression due to its widespread use. However, most of the concepts discussed apply more generally to networks derived from other metrics.

In addition to how co-expression is quantified between input genes, different approaches can be used to define the starting source when examining co-expression relationships among genes. The simplest approach is to target a set of guide genes which are simply the subset of genes an investigator is interested in. Co-expression is calculated for each guide gene and interactions are identified on a pergene basis, usually using a hard correlation cut-off (e.g. $r^{2}>0.9$ ). This contrasts an all genes approach, which involves exhaustively computing interactions among all pairwise combinations of genes to construct a global context for gene co-expression [25]. After a global network is constructed, significant interactions are identified by examining the overall distribution of correlations within the network. With the onset of high throughput sequencing technologies, leveraging whole genome comparisons of expression profiles has become the standard approach for analyzing co-expression relationships. Given hundreds of millions of potential interactions among tens of thousands of genes, it is critical to establish a robust statistical framework to evaluate the significance of co-expression interactions, defining which interactions are statistically significant to differentiate true co-regulatory relationships from gene pairs that simply randomly exhibit similarity.

\subsubsection{Interaction transformation and normalization establish a statistical background for significance}

An interaction between two genes using one of the metrics discussed in section 2.1 quantifies the similarity between two genes' expression profiles. Most co-expression network applications make an assessment of statistical significance on each pairwise similarity in order to define a network. For example, Usadel et al. leveraged the fact that a correlation coefficient is t-distributed for small $\mathrm{N}$ to directly assign $p$-values to co-expression relationships [26,27]. Others have applied a Fisher transformation to the correlation coefficient $[2,8,9,11,28-30]$, which results in a normal sampling distribution whose variance is related to the length of the expression profiles [31]. This establishes a convenient null expectation that a dataset containing no biological information will be normally distributed with a defined variance that relates to the size of the vectors being correlated. Deviations from this distribution are thus considered to be potentially interesting biological signal.

In addition to establishing a statistical interpretation for interaction scores, statistical measures of significance also facilitate proper thresholding of co-expression networks. Gene co-expression measures are often calculated for each pairwise combination of genes, resulting in a complete gene-by-gene matrix of co-expression scores. However, it is not until a threshold has been established and insignificant interactions are removed that a network arises. Simple approaches such as defining a global threshold on similarity scores (e.g. $Z \geq 3$ ) to binarize interactions quickly provides interpretable networks. While a binarized approach is simple to implement, it requires the investigator to define a hard, global threshold, the position of which can be arbitrary and does not necessarily reflect the continuous nature of the underlying co-expression information [32]. Other methods retain interaction weights. In several 
studies, Fisher-transformed PCCs derived from a particular expression dataset are then standard normalized, and the resultant interaction scores for co-expression among two genes can be interpreted directly as the number of standard deviations (Z-score) a given interaction is from network background $[9,29,33]$. Other approaches directly transform the similarity matrix, converting values to a weighted adjacency by raising the matrix to a power $(\beta \geq 1)$, emphasizing stronger interactions in the network [32].

By definition, thresholding requires the investigator to specify a cutoff. However, prior knowledge can be used to inform this decision. Network analyses across many fields have found that, in naturally arising networks, the degree distribution of interactions across nodes often follows a power-law distribution, an occurrence called a scale-free network [34]. This emergent property of biological as well as other naturally occurring networks suggests that networks exhibit a small number of "hubs," which are nodes with very high connectivity, compared to most nodes, which have a small number of interactions. Using this expectation of how network interactions should be distributed, previous work has suggested that informative thresholding cutoffs can be established by checking for a goodness of fit to the expected power law distribution [35]. However, the assumption that all biological networks strictly follow a power law distribution has also been questioned by several studies [36,37], and acceptable alternatives such as the truncated power law or other heavy-tailed distributions have been proposed [3]. Nonetheless, matching the empirical degree distribution against theoretical expectations to establish a reasonable cutoff provides a complement for other approaches and directly relies on statistics at the level of individual gene pairs.

\subsection{Extracting functionally coherent modules from co-expression networks}

Biological processes are thought to be carried out by modules of interacting gene products [15]. Genes that exhibit similar patterns of co-expression across a diverse set of accessions or experiments have an increased likelihood of being within the same functional module based on the principle of "guilt by association" [38] (Figure 2). Several methods exist to assess the level of biological information present within a co-expression network. One approach is to test for enrichment of significant co-expression among functionally annotated sets of genes as defined by curated functional standards, which can both serve as a benchmark to measure how much known information is potentially captured by the network as well as a method to annotate clusters of genes. Other methods do not rely on prior functional annotation, focusing instead on network structure. Especially in agricultural species, where definitive functional annotations are still not available for most genes, simple cluster analysis can provide insight into the biological coherence of highly co-expressed genes. The remainder of this section focuses on functional evaluation of co-expression networks using gene annotations as well as systematically evaluation biological coherence of network clusters, even in the absence of prior knowledge. 


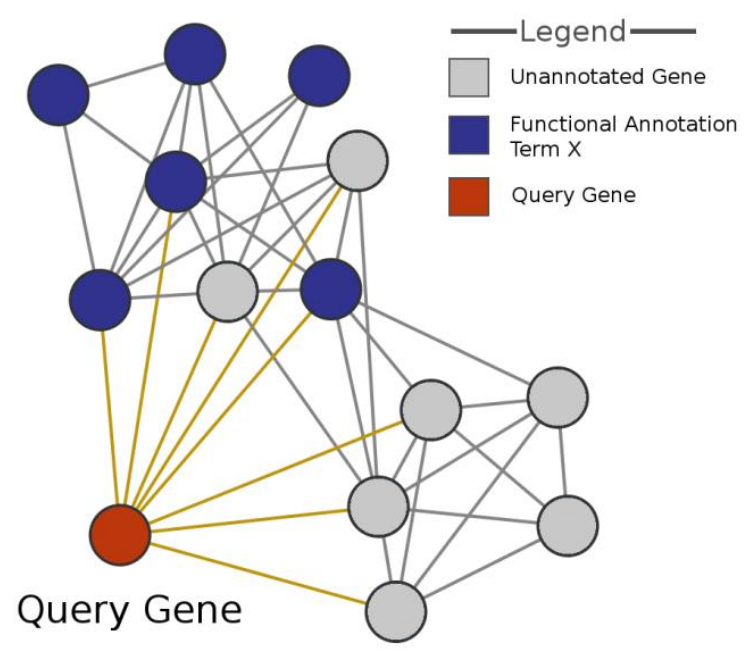

Figure 2

Assessing gene function through guilt by association. A query gene (red node) is used to identify gene modules with strong co-expression (yellow edges). Neighborhoods include clusters of genes with no previously annotated function (gray nodes) as well as clusters of genes enriched for functional term "X" (blue nodes; Section 2.2.1). Putative function can be assigned to each cluster (Section 2.2.2) using the concept of guilt by association.

\subsubsection{Ontology Enrichment}

Work on characterizing gene function in plants has led to the development of curated, functional ontologies such as the Gene Ontology (GO) [39], the Kyoto Encyclopedia of Genes and Genome (KEGG) [40], and MAPMAN for genes involved in metabolic pathways [41]. These resources can serve as a benchmark for how well gene co-expression networks are able to characterize already known functional relationships among genes co-annotated within these ontologies [42]. This approach has been used to validate co-expression networks in agricultural crops including barley [43] and maize [9] and has been extensively applied in model organisms [44]. Enrichment for genes annotated to particular ontological terms is tested under the null expectation that co-expression among gene co-annotated to an ontology term should be no different than the background co-expression level [45]. Significant enrichment of coexpression among genes previously annotated for functional terms establishes a high level view into which processes are effectively 'captured' by a network providing an overall benchmark of how many ontology terms are significantly co-expressed compared to a random expectation. This enrichment of co-expression among genes with well-established functional relationships bolsters confidence that network clusters, annotated or not, are likely to contain useful information. Previously annotated genes that are strongly co-expressed with a query gene or a set of un-annotated genes can be used to assign putative function, but also provides an opportunity to assign putative function to unannotated clusters. (Figure 2). Furthermore, it is likely that there exist sets of genes that exhibit strong co-expression, and possible biological coherence, that currently lack any known functional annotation (Figure 2; gray nodes). Given proper experimental design, highly co-expressed clusters of genes lacking known annotations still likely contain useful information. While enrichment of previously characterized functional terms can bolster confidence that a network contains biologically relevant information, it fails to further describe processes that are less studied or yet to be discovered. 


\subsubsection{Clustering}

Complementary to approaches that leverage previously curated functional annotations, unsupervised approaches such as network clustering provide novel insight into biological processes captured within co-expression networks. Sets of genes that exhibit a high degree of co-expression with each other can be systematically identified within a co-expression network using several different approaches. Genes can simply be assigned to clusters using any standard clustering algorithm (e.g. hierarchical agglomerative or k-means clustering) by analyzing the gene expression profiles themselves. However, these approaches do not leverage the full potential of network structure. Graph clustering approaches can operate directly on the network, which may provide a more direct view of structure represented by co-expression relationships. For instance, the Markov Cluster Algorithm simulates random walks on the network, where clusters are defined by the frequency of paths connecting between genes [46]. Another commonly used tool, Weighted Correlation Network Analysis (WGCNA) takes a hybrid approach. First, it calculates gene-gene proximity values using a method called topological overlap measure (TOM), then defines gene clusters by hierarchically clustering the gene-gene proximity matrix [32]. Another approach called Improved Principle Component Analysis (IPCA) [47] was used by Fukushima et al. to define over 400 gene clusters from a co-expression network in tomato from which $88 \%$ of the clusters were enriched with co-annotated genes from GO terms [2]. Schaefer et al. clustered co-expression networks using both $\mathrm{MCL}$ as well as a simpler approach where clusters were identified visually after the network was displayed using a force-directed layout algorithm. The authors found substantial overlap between clusters identified visually and those identified through MCL [9].

The wide scope of available methods to discover clusters within a network coupled with the directed approach of examining clusters for known functional enrichment provides a rapid way to both validate existing co-expression networks as well as to analyze uncharacterized interactions for novel biological meaning.

\section{Current applications of co-expression networks in crops}

Historically, large scale microarray experiments have extensively been used to characterize coexpression networks in the plant model Arabidopsis thaliana [48]. Broadly, experiments surveyed genome-wide expression response to abiotic stress [49], plant tissue and development [10] as well as response to different plant hormones [7]. In general, analysis of patterns of gene expression identified an enrichment for co-expression among genes that are co-annotated in both KEGG terms [50] as well as the Gene Ontology [10]. These proof of principle approaches blazed in model systems naturally extend to analogous experiments performed in agricultural species.

To date, gene co-expression networks have been used to rapidly predict gene function in many nonmodel plant species, many of which have agricultural importance. Most networks were built with the purpose of discovering and characterizing highly connected subnetworks or modules to better understand various phenotypes or functions or to provide a general resource to the community. Such studies have now been completed for a variety of agronomic species including as soybean, poplar, grape, alfalfa, rice, maize, tomato, and barley [43,51]. A typical study involves using either publicly or self-generated expression data to build a network, looking for functional enrichment within modules, then focuses directly on a module of interest for biological interpretation.

Changes in gene expression can occur from introducing variation stemming from many different sources. As with model species, this variation can be examined in the context of co-expression networks to assess the putative functional impact of different experimental conditions in crop species. Recent studies performed in crops generally survey gene expression variation that arises from several major 
sources reviewed here: changes in environmental conditions; developmental and organ based variation; and variation due to population and ecological dynamics.

\subsection{Co-expression in response to environmental conditions}

Co-expression networks can be built with the intent of discovering various gene modules that respond to environmental conditions. Factors such as diseases and/or abiotic stressors can have a very broad impact on the development and phenotype of a plant, causing expression profile changes that differ among individuals with different genetic variation or those exposed to different environmental conditions. Co-expression networks can be built by measuring transcriptomic changes under these conditions to generate environmental specific co-expression networks $[52,53]$. For example, Mochida et al. assembled a global co-expression network from 1,347 experiments surveying both diverse environmental conditions and stresses in barley [43]. They discovered functional modules using gene ontology enrichment as well as Triticeae-specific network modules using comparative approaches.

Zheng et al. studied citrus response to one of its most destructive diseases, Huanglongbing, more commonly known as citrus greening [53]. Using various transcriptome datasets and a set of genes that are up-regulated in early stages of inoculation, they were able to construct and identify several modules that provided insights into the mechanism of immune response to citrus greening. Sarkar et al. studied rice's response to heat stress discovering various genes/modules that could help provide insight in mechanistic changes in response to stress [52]. Gene expression profiles from rice exposed to two different durations of heat stress were used to discover several modules consisting of functionally correlated genes, which included previously documented genes involved in heat stress.

\subsection{Tissue and developmental atlases for agricultural crops}

Different developmental time points and/or tissues have also been used to build and study modules within a co-expression network. Just like different environmental conditions, different developmental time points and/or tissues can have varying expression patterns. Expression data from these categories have been applied to a variety of agricultural crops to look for tissue enriched modules [1,9,53-57]. As an example, Ozaki et al. built a tomato network using microarray data and discovered that 75 out of their 199 modules had significant functional enrichment [58]. They further investigated a module related to the flavonoid biosynthetic pathway and compared genes within that module to a transgenic cultivar identifying genes that were up-regulated in flavonoid biosynthesis.

Downs et al. and Sekhon et al. sampled the expression of different tissue types and developmental stages to discover tissue enriched modules $[8,11]$. Downs et al. used maize tissue from 50 different developmental time points to build developmental co-expression networks. Using those tissues, they were able to discover 24 modules where subsets of genes were associated with specific tissues, or different developmental stages of a tissue. Sekhon et al., similarly, surveyed 60 tissue/time points and found that many genes displayed organ specific expression patterns [11]. Schaefer et al. leveraged coexpression networks to identify general functional modules derived from both developmental as well as genotypic diversity. They built networks using publically available developmental and genotypic expression data in maize to not only show functional enrichment within modules, but also to demonstrate differences across networks as a way to capture biological function.

\subsection{Comparative based co-expression approaches}

Co-expression networks can also be built with the purpose of discovering conserved modules across species. Even though the majority of genes for agronomic species do not have a functional annotation, investigators can use modules composed of well-defined, functionally annotated genes from one species to compare and discover similar modules in another. These comparisons are not limited to using co- 
expression networks from model species such as Arabidopsis [59-62], but can be applied using other species if they are using genes with well-defined functional annotations $[30,63]$.

Obertello et al. built rice and Arabidopsis nitrogen regulatory networks to discover genes that are directly related to nitrogen use. They first identified genes that were induced or repressed by nitrogen regulators using both rice and Arabidopsis expression data. Conserved, nitrogen-related gene clusters and predicted transcription factors were identified by creating a cross-species functional network that combined putative protein-protein and regulatory interaction from both rice and Arabidopsis, including data from gene expression, using orthologous genes to share interaction evidence across species. Similarly, Itkin et al. used comparative co-expression network analysis to identify genes related to steroidal glycoalkaloids (SGA), a toxic substance found in some tubers and tomatoes [63]. Using homologs (SGT1/GAME1) in tomato and potato as seed genes, the authors discovered highly coexpressed genes in each species that are involved in SGA biosynthesis. Targeting these co-expressed genes, they were able to successfully reduce SGA concentrations in both species using virus-induced gene silencing.

Evolutionarily conserved functional modules can also be discovered by integrating a wide variety of species specific co-expression networks [60]. More recently, Ruprecht et al. used multiple plant species together to discover a wide variety of conserved modules, with an emphasis on modules related to cell wall formation [64]. In a similar approach, Leal et al. used gene expression data from Arabidopsis, Maize, soybean, rice, tomato and cassava to identify conserved genes involved in immune response under pathogenic stress [62]. Using the networks that were built from the expression data, they were able to find functional similarities in the immune response across species.

Co-expression analysis has also been leveraged to gain meaningful insight between domesticated agricultural species and their wild relatives. For example, Swanson-Wagner et al. used 38 diverse maize genotypes and 24 teosinte genotypes to generate separate co-expression networks for maize and teosinte, its wild relative. They were able to identify gene clusters that were rewired between maize and its crop wild ancestor, suggesting modulated regulation could have played a role during domestication [30]. The genes identified by this differential co-expression analysis complemented those identified through standard differential expression analysis. Cho et al. used transcriptome and metabolite data to profile sprouts from three evolutionarily divergent potato cultivars to characterize genes involved in anthocyanin production. Comparing these data, they were able to find 119 genes that were strongly correlated with anthocyanin-related metabolites [65].

\section{Important considerations for constructing and interpreting co- expression networks in the context of crop species}

Most studies that focus on co-expression analysis use slightly different tools and methods, but the core concepts are quite similar. Most of the well-established tools for co-expression networks were originally developed for data-rich model systems, and for the most part, these tools have been successfully translated to non-model systems. However, many non-model systems, including crops, require additional considerations before meaningful results can be derived from co-expression networks. For example, differences in sequencing platforms or qualities of reference genomes fundamentally alter how gene expression is measured for a given species. In this section, we discuss several important technical and biological themes that should be considered when applying co-expression network analysis outside the context of model species. 


\subsection{Transitioning from array based expression to next generation sequencing technology}

Technology for measuring gene expression has matured over the past decade and can now produce highly quantitative measurements of expression on a genome scale for relatively low cost. Traditional, array-based methods typically measure transcript levels by quantifying the amount of sample RNA that hybridizes to probes that are physically attached to a glass slide [66]. Examining the RNA transcripts of a cell using array-based approaches has specific limitations, mainly having a limited detectable range of expression and a reliance on an existing genomic sequence, which until recently, has been a challenge in agricultural species [67].

In many crop species, next generation RNA-Seq technology is the only platform that is available due to draft-quality reference genomes, making it difficult to design high quality probes. Next generation sequencing technologies enable de novo sequencing of transcripts using RNA-Seq technology making it possible to combine draft reference genome contigs with de novo assembled contigs discovered through transcriptome assembly [68-70]. RNA is isolated from tissues of interest are converted into cDNA libraries with adapters ligated to either end of the molecule. The CDNA is generally sequenced from both ends, resulting in short, 'paired end' reads that are mapped to the reference genome (or assembled de novo) together with the expected DNA fragment length between them. Mapped reads are normalized by the total read amount to produce a 'digital' expression value in fragments per kilobase per million reads (FPKM) [67].

As more focus is put on the latest generation of sequencing technologies, it is important to consider how changes in platform might affect results or potential biases that could be introduced. In the context of co-expression, a study in maize compared co-expression networks from the same tissues constructed from both RNA-Seq and microarrays in identical 60 developmental tissues [57]. They showed that a high correlation (Pearson correlation 0.70-0.83) existed between co-expression interaction matrices as well as nearly identical clustering of tissues based on hierarchical clustering, indicating that networks derived from both technologies are robust and reproducible.

Differences between microarray and RNA-Seq were also found. RNA-Seq provided a higher resolution of detection for paralogous genes as well as enhanced coverage over the genome, as it did not rely on probes designed from gene models. Furthermore, differences in dynamic ranges between microarrays and RNA-Seq led the authors to utilize slightly different raw expression transformations [57]. The authors reported genes that exhibited substantial differences in their co-expression relationships between the two platforms were enriched for genes with low expression. Many of these genes exhibited what appeared to be spurious high correlations resulting from limited coverage in the RNA-Seq data, suggesting that some caution should be taken when interpreting co-expression relationships for lowly expressed genes derived from both RNA-Seq and microarray technology.

\subsection{Generating co-expression networks from genotypically diverse transcriptional data}

The transition from array based techniques to NGS based expression studies poses several other interesting questions, especially in crop species for which many have only recently had an available reference genome sequenced. Higher throughput technologies have enabled more elaborate comparative studies that survey genetic diversity of populations as well as the ability to detect the effects of larger structural variation of insertions and deletions and copy number variation [4]. This ability to survey diverse genomes also comes with difficulties. For example, where the maize and human genomes are comparable in size, on average, two maize lines contain an order of magnitude more variation than any two humans [71]. This high level of diversity can dramatically increase the number of mismatches in reads from diverse individuals when mapping to the reference transcriptome, which reduces the number of reads that map correctly [4]. It can be difficult to discern sequencing errors from 
true sequence variation[72]. These discrepancies may have a significant impact on the ability to reliably quantify RNA-Seq reads, consequently impacting measurement of gene expression levels due to a lack of read.

This natural genotypic (and subsequently phenotypic) variation between individuals within a species is represented both by a 'core' genome which is genomic content shared by all individuals within a species as well as a 'dispensable' genome which constitutes genomic content only found in some individuals. Together, the core and dispensable genome, observed across a population of individuals, represent the pan genome [73]. The pan-genome exacerbates this issue of reliably measuring gene expression due to the fact that when mapping reads to the reference genome, it becomes exceedingly difficult to differentiate between low expression of spuriously mapped reads to portions of the dispensable portion of the pan genome, essentially mis-quantifying structural variation or presence/absence variation (PAV) that can be extensive even between two lines within a species [74-76]. Standard data analysis workflows often model approaches in species with more definitive reference genomes or less genotypic variation, leading to a bias toward genes that exist in the core genome. However, as discussed above, methods are available to assemble transcriptomes de novo, which lead to a better representation of the pan genome. For example, Hirsch et al. identified over 31,000 additional transcript assemblies from unmapped RNA-Seq reads in their analysis of the maize pan genome. De novo assembly, however, comes with its own unique challenges and caveats. It is often difficult to distinguish transcripts that originate from alternative splice forms, paralogs, homologs, and homeologs. After extensive cross species and comparative analysis, Hirsch et al. retained just over 8,000 high confidence transcript assemblies that were absent from the core maize reference genome.

In the context of co-expression, presence/absence variation detectable using de novo assembly can cause issues when measuring correlation coefficients, which do not handle missing data well and are sensitive to artificially low expression levels that result from read mapping issues. We have previously explored methods to detect false positive co-expression clusters that are driven by artificially high or low expression due to presence/absence variation in a subset of genotypically diverse accessions [9]. We found that 2 of 21 global co-expression clusters had a statistically significant overabundance of known copy number variant (CNV) genes indicating that clusters potentially driven by PAV exist, but no indication that they are pervasive. To our knowledge, no methods have been proposed to remove these interactions. Despite the challenge of quantifying expression in species having diverse genomes, coexpression has been extensively used to characterize biological function in crop species using several different approaches.

\subsection{Network Context Matters}

Once a network has been built and validated to contain enrichment for known biological information, focus switches to examining interactions among candidate genes of interest. Popular approaches include using a single or small set of genes as 'bait' and extracting genes that are significantly coexpressed with the input set $[9,25,38,42,77,78]$. Unraveling the putative functional processes of genes with unknown function based on guilt by association is dependent on which neighboring genes it is coexpressed with. However, as we show here, co-expression interactions measured in a given network can heavily rely on the type of expression data that is used to build the network.

Figure 3 shows average expression profiles from 19 co-expression clusters identified within a coexpression network built from 60 different maize developmental tissues derived from the reference line B73 (Figure 3A) compared to 21 clusters identified in a co-expression network built from 62 genotypically-diverse whole seedling samples (Figure 3C) (Figure modified from [9]). Each column of the heatmap plots the average normalized expression profile across the entire set of samples. The patterns 
driving co-expression for clusters in each network are fundamentally different. Clusters in the developmental network are largely driven by increased or decreased relative expression across several tissues. In contrast, clusters in the genotype network are often distinguished by under-expression in a single or small number of diverse maize accessions (with the exception of cluster ' $l$ ', which has consistent over-expression in a single accession). These fundamental differences in co-expression patterns ultimately highlight regulatory relationships for different biological processes. For example, Figure 3B plots a mean co-expression interaction score for genes co-annotated to specific Gene Ontology terms in each network (See [9] for implementation details), contrasting the signal in the two networks for each term. While a large number of biological processes (red) are significantly coexpressed $(Z \geq 3)$ in both networks, there are a substantial number of terms that are only significant in either the genotype network (green) or the developmental network (blue). The fundamentally different patterns of gene co-expression coupled with different representation of co-expressed Gene Ontology terms indicates that the expression context from which a gene co-expression network is constructed matters. The expression context directly affects which genes' expression vary, and consequently, what biological processes are effectively captured within a network, substantially impacting interpretability of results for a given hypothesis.
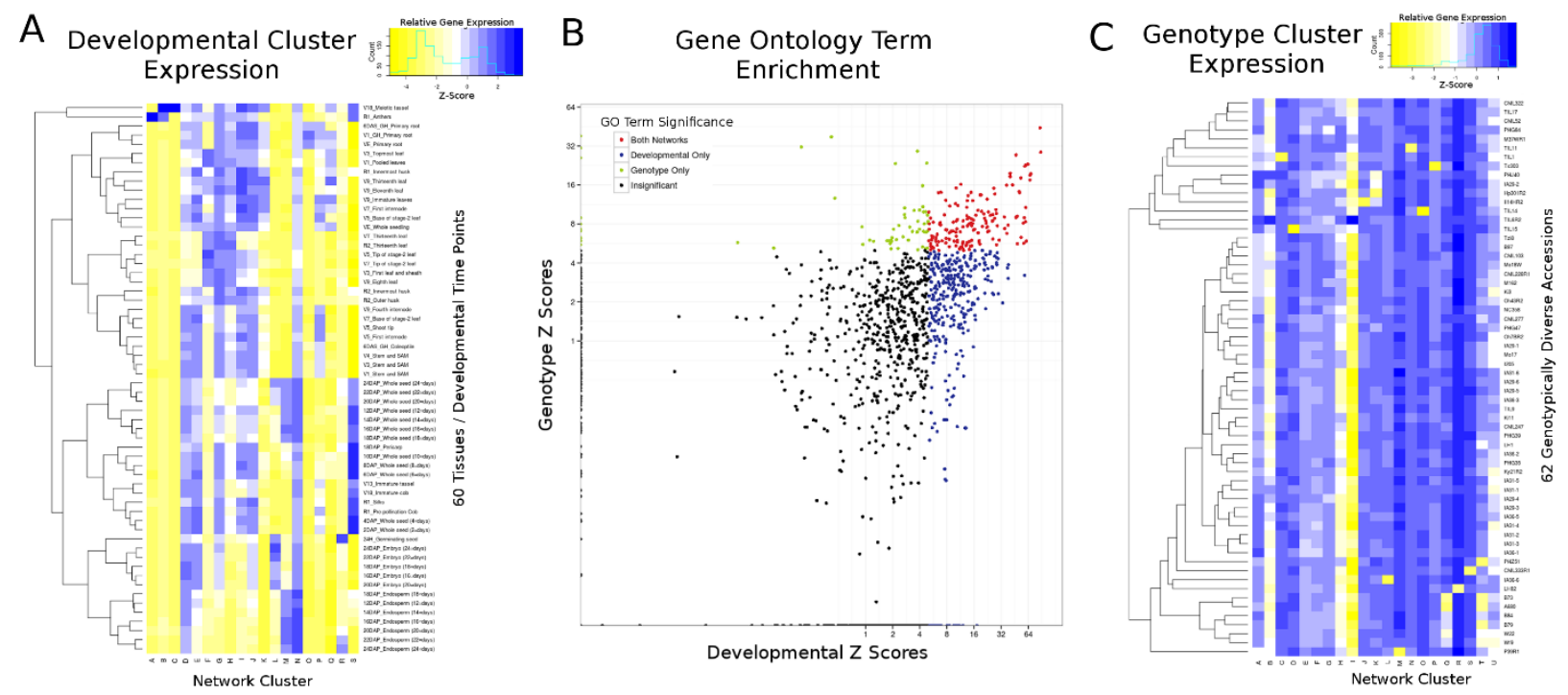

Figure 3

Expression context drives different network structure in developmental versus genotypic variation. Two gene co-expression networks were constructed from whole genome transcriptional data derived from different sources. Both networks were clustered and average gene expression profiles were calculated for each cluster. (A) Average, representative gene expression profiles are shown for network clusters across 60 different maize tissues and developmental timepoints. Clusters generally exhibit high levels of expression in a small subset of tissues. (C) Average, representative gene expression profiles shown for network clusters from whole seedling RNA isolated from 62 genotypically diverse maize genotypes. Clusters generally exhibit patterns of low expression in a single or small number of genotypes. (B) Comparative Z-scores of co-expression among genes co-annotated to Gene Ontology (GO) terms (points) plotted on a log-log scale. Significant co-expression ( $Z \geq 3$ ) observed for $G O$ terms only in the genotype network are shown in green, developmental network only in blue and significant in both networks in red.

Where co-expression networks in model species are often constructed from genotypically homogenous individuals surveyed in different tissues, conditions, or timepoints, many questions in agricultural species are centered around questions of genotypic or phenotypic diversity. Many of the interesting phenotypes in agricultural species arise from different sources of variation: standing genotypic diversity 
within a population, a plant's temporal and spatial development cycle, and the plant's interaction with the environment. Due to the disparate expression patterns that drive co-expression among these different sources of biological variation, networks must be constructed from a context or set of samples that reflect the variation targeted by the investigator. For example, it is likely that a co-expression network derived from a tissue or developmental atlas in a reference individual will not capture relationships for genes in processes that underlie phenotypic variation across genotypically diverse individuals.

\section{Looking forward: emerging applications of co-expression network analysis \\ 5.1 Co-expression networks in the context of association studies}

High throughput sequencing technologies are now being readily applied to many agricultural species. The availability of these sequencing technologies has accelerated the rate at which we are able to survey gene function on a genome wide level. In addition to gene expression profiling, other methods have been used to associate genetic variation with agronomic traits of interest. Current approaches typically involve mapping agriculturally important traits using forward genetics approaches such as quantitative trait loci (QTL) mapping and, more recently, genome wide association studies (GWAS). Particularly in agricultural plant species, the ability to make directed crosses to construct high resolution mapping populations has made association mapping the standard approach in examining standing genetic variation and its phenotypic consequences in many agricultural species [79].

While fundamentally different in their approach, both GWAS as well as co-expression relate genes that putatively occur within the same biological process. GWAS statistically associates genetic variation with traits of interest where gene co-expression networks identify sets of genes which are co-regulated and often functionally coherent. If genetic variation driving phenotypes identified by GWAS is encoded by co-regulated genes, the loci that are labeled as putatively functionally related by each approach should overlap in the genes they identify.

Having a tremendous amount of statistical power derived from targeted mapping populations [79], well designed GWA studies can sometimes find hundreds of loci or single nucleotide polymorphisms (SNPS) that are strongly associated with a trait of interest. Despite the ability to statistically associate many loci with a trait of interest, the causal genes and functional alleles remain unknown in many cases, even for successful GWAS. Associated SNPs are likely in linkage disequilibrium (LD) with functional alleles. The extent at which LD decays varies across the genome [80] as well as between species [71], however LD can also vary substantially depending on whether the genetic material being analyzed is a landrace or cultivated, outcrossing or inbreeding $[81,82]$.

Particularly in agricultural species, these issues severely hamper the interpretation and narrowing of large candidate gene lists using positional and/or functional information, especially when the study includes populations with diverse haplotype structure with varying levels of LD at any given locus $[81,82]$. This candidate resolution issue is exacerbated even further by cases where trait associated SNPS do not resolve to genic regions, which can account for nearly half of significantly associated SNPs and usually enriched within a $5 \mathrm{~kb}$ region upstream of genes $[83,84]$. Even using straightforward SNP-to-gene mapping rules, such as the nearest two genes (upstream and downstream candidates), identifying the true functional gene at each locus for complex traits involving potentially dozens of significantly associated SNPs quickly becomes unwieldy, potentially doubling, or worse, the number of potential candidate genes from candidate SNPs upfront. With little or no functional information, this expansion in 
the number of possible candidate genes can potentially result in a very high false discovery rate when determining the causal locus for the trait of interest.

Even when high confidence candidate genes can be identified, functional, mutant or transgenic based validation experiments on a large scale outside of model species are cumbersome and expensive to perform. However, in the absence of mutant analysis, co-expression among genes near significant GWAS SNPs are an informative and easily measurable source of functional data. With the adoption of next generation sequencing, whole-genome expression studies are now surveying agricultural transcriptomes in large numbers, which could complement current functional association studies in crops. Schaefer et al. built co-expression networks from publically available expression datasets to examine interactions among genes near SNPs identified by a leaf architecture GWAS [9,85]. Direct integration of significantly associated GWAS SNPs with expression based functional networks provides a potential approach for rapid prioritization of interesting candidate genes with enough functional support to justify investing resources for mutant or transgenic validation.

One potential drawback of this approach is that there is no guarantee that GWAS candidate genes will be significantly co-regulated in any given co-expression network functional context. Networks constructed from different types of gene expression data represent different biological processes to varying degrees. It is possible that co-regulatory relationships between genes identified by GWAS are in a network that has yet to be generated.

Future investigation will be necessary into understanding how these types of complementary data relate to each other as well as the development of formal methods to facilitate this integration. Additionally, many breeding phenomena, such as heterosis, to our knowledge have been wholly uncharacterized in the context of co-expression bases approaches. As the discovered number of loci associated with traits increases, it will become important to characterize how genes work together to affect phenotypes of interest.

\subsection{Higher resolution tissue maps and single cell sequencing technology}

Widely used plant tissue atlases survey gene expression among larger tissues and incorporate expression changes across developmental time points and are continuously increasing resolution by incorporating additional tissues or developmental stages $[13,33,57,58,86]$. As can be seen in Figure $3 \mathrm{~A}$, patterns of co-expression derived from global tissue atlas datasets are largely driven by consistent up or down expression in a small sets of tissues, which are often physiologically related. For example, clusters A, B and C are driven by overexpression in the tassel, the anthers, or in both. This tissue specific coexpression structure can be further broken down into higher resolution tissue maps with more focused expression profiling of a set of closely related tissues, for example, using targeted approaches such as tissue laser-capture microdissection (LCM) that can generate mRNA from as little as 1000 captured cells [87].

Such approaches have been used to examine fine level expression patterns in 6 stages of maize shoot apical meristem development [88] as well as 9 targeted tissues of maize endosperm [89]. While currently, these sample sizes are not adequate to capture enough variation to build robust coexpression networks, they can be integrated or compared with other high resolution tissue samples to examine finer scale tissue structure. This additional resolution will help distinguish tissue specific relationships, ultimately leading to more informative functional maps built upon co-expression interactions.

As sequencing costs continue to decline and technological advances make higher resolution tissue atlases possible, we envision an expanded atlas with an added component of genetic diversity. As most 
tissue atlases target a single genotype, biological processes that vary across populations will be missed without measuring tissue-specific expression across genotypically diverse individuals. Complementary to integration with GWAS studies discussed above (which also rely on differences in genotype) tissue specific, genotypically diverse co-expression networks could potentially be the next step forward in further unraveling gene function for important traits both in crops and other species.

\subsection{Systematic integration of heterogeneous whole genome functional data}

While co-expression provides a rich source of functional information, not all biological processes can be fully captured by co-regulation relationships reflected in gene expression profiles. There exist several other data types that can be quantified on a whole genome level. Metabolite abundances have been used to build co-metabolite networks in tomato as an effective method to identify metabolite modules [90]. Methods that effectively bridge connections across transcription, metabolites and other layers of biological information will be a powerful approach to explaining the relationship between genotype and phenotype [91].

A recent study in potatoes showed links between metabolites linked to anthocyanin production in three different colored potatoes via differential expression among genes known to play roles in flavonoid production, hormone metabolism, regulation of transcription and cell signaling [65]. Robustly integrating several types of heterogeneous functional data such as co-expression with co-metabolite networks may alleviate instances where the expression variation was not measured in the ideal network context. For example, incomplete co-expression interactions among genes related to biological processes (e.g. anthocyanin production) could be bolstered by interactions among metabolites known to be related to certain pathways, effectively recovering network interactions missed by transcriptional variation.

In addition to expression based functional networks, we anticipate that other rich forms of functional expression data such as protein abundance and metabolite levels will play an important role in characterizing the link between genotype and phenotype.

\subsection{Co-expression outside of agricultural species}

The adoption of network based, systems biology approaches to understand gene function is not limited to agricultural species. Already, co-expression based approaches are being applied in fields now positioned to leverage next generation sequencing technologies such as ecology [92], fisheries and wildlife [93] or evolution [94]. Co-expression also can be used to study polyploidy plants as well as the conservation and functionalization of duplicated genes (Lin Li, personal communication; [95]) and gene families [10]. As sequencing costs continue to drop and methods for analysis become more mature outside of model systems, the impact of gene functional networks derived from co-expression analysis will facilitate tests of long standing hypotheses on how genes function as units in all species [96].

\section{Conclusions}

It is an exciting time for agricultural research. The ability to rapidly measure global gene expression on a large enough scale to create robust, descriptive co-expression networks is a major leap forward for many species, particularly non-model systems that, until recently, had modest genetic tools. As reviewed here, co-expression based approaches to infer gene function are already well-utilized in many non-model species and being used to describe how genes putatively interact to perform biological processes that impact important agronomic traits.

Since the first genomes were sequenced 20 years ago, massive amounts of resources have been invested in the ability to globally survey genomes and transcriptomes. The bottleneck in discovery has 
quickly shifted from being able to generate genome-wide data to being able to meaningfully interpret it. With each new species comes unique biological and methodological considerations. As newer technologies allow for more precise measurement of both transcriptional data as well as other forms of rich functional data, interdisciplinary approaches and collaboration between experimental and computational biologists will be more important than ever. Additionally, as tools to create and analyze co-expression networks mature, publically available datasets in easily usable forms will remain even more important to enable functional profiling of genes across many different experimental contexts. Unraveling gene function using holistic, integrative systems biology approaches will certainly impact our ability to increase agricultural output and to address problems with global food security looming on the horizon.

\section{Acknowledgements}

The authors would like to thank Ivan Baxter and Brian Dilkes for their constructive comments and insight in the writing of this manuscript.

\section{Financial Support}

RJS and CLM were partially supported by Grant DBI-0953881 and Grant IOS-1126950 from the National Science Foundation. CLM is partially supported by the CIFAR Genetic Networks Program. RJS is supported by Grant 2016-67012-24841 from the National Institute of Food and Agriculture. JMM is supported by a Biomedical Informatics and Computational Biology (BICB) Graduate Fellowship.

\section{References}

[1] C.M. Andorf, E.K. Cannon, J.L. Portwood, J.M. Gardiner, L.C. Harper, M.L. Schaeffer, B.L. Braun, D.A. Campbell, A.G. Vinnakota, V. V Sribalusu, M. Huerta, K.T. Cho, K. Wimalanathan, J.D. Richter, E.D. Mauch, B.S. Rao, S.M. Birkett, T.Z. Sen, C.J. Lawrence-Dill, MaizeGDB update: new tools, data and interface for the maize model organism database., Nucleic Acids Res. 44 (2016) D1195-201. doi:10.1093/nar/gkv1007.

[2] A. Fukushima, T. Nishizawa, M. Hayakumo, S. Hikosaka, K. Saito, E. Goto, M. Kusano, Exploring tomato gene functions based on coexpression modules using graph clustering and differential coexpression approaches., Plant Physiol. 158 (2012) 1487-502. doi:10.1104/pp.111.188367.

[3] S.H. Strogatz, Exploring complex networks., Nature. 410 (2001) 268-76. doi:10.1038/35065725.

[4] P.L. Morrell, E.S. Buckler, J. Ross-Ibarra, Crop genomics: advances and applications., Nat. Rev. Genet. 13 (2011) 85-96. doi:10.1038/nrg3097.

[5] M.B. Eisen, P.T. Spellman, P.O. Brown, D. Botstein, Cluster analysis and display of genome-wide expression patterns., Proc. Natl. Acad. Sci. U. S. A. 95 (1998) 14863-8. doi:10.1073/pnas.95.25.14863.

[6] Y.X.R. Wang, M.S. Waterman, H. Huang, Gene coexpression measures in large heterogeneous samples using count statistics, Proc. Natl. Acad. Sci. 111 (2014) 16371-16376.

doi:10.1073/pnas.1417128111.

[7] H. Goda, E. Sasaki, K. Akiyama, A. Maruyama-Nakashita, K. Nakabayashi, W. Li, M. Ogawa, Y. Yamauchi, J. Preston, K. Aoki, T. Kiba, S. Takatsuto, S. Fujioka, T. Asami, T. Nakano, H. Kato, T. Mizuno, H. Sakakibara, S. Yamaguchi, E. Nambara, Y. Kamiya, H. Takahashi, M.Y. Hirai, T. Sakurai, K. Shinozaki, K. Saito, S. Yoshida, Y. Shimada, The AtGenExpress hormone and chemical 
treatment data set: experimental design, data evaluation, model data analysis and data access., Plant J. 55 (2008) 526-42. doi:10.1111/j.0960-7412.2008.03510.x.

[8] G.S. Downs, Y.-M. Bi, J. Colasanti, W. Wu, X. Chen, T. Zhu, S.J. Rothstein, L.N. Lukens, A Developmental Transcriptional Network for Maize Defines Coexpression Modules, PLANT Physiol. 161 (2013) 1830-1843. doi:10.1104/pp.112.213231.

[9] R.J. Schaefer, R. Briskine, N.M. Springer, C.L. Myers, Discovering functional modules across diverse maize transcriptomes using COB, the Co-expression Browser., PLoS One. 9 (2014) e99193. doi:10.1371/journal.pone.0099193.

[10] M. Schmid, T.S. Davison, S.R. Henz, U.J. Pape, M. Demar, M. Vingron, B. Schölkopf, D. Weigel, J.U. Lohmann, A gene expression map of Arabidopsis thaliana development., Nat. Genet. 37 (2005) 501-6. doi:10.1038/ng1543.

[11] R.S. Sekhon, H. Lin, K.L. Childs, C.N. Hansey, C.R. Buell, N. de Leon, S.M. Kaeppler, Genome-wide atlas of transcription during maize development., Plant J. 66 (2011) 553-63. doi:10.1111/j.1365313X.2011.04527.x.

[12] P.T. Spellman, G. Sherlock, M.Q. Zhang, V.R. Iyer, K. Anders, M.B. Eisen, P.O. Brown, D. Botstein, B. Futcher, Comprehensive identification of cell cycle-regulated genes of the yeast Saccharomyces cerevisiae by microarray hybridization., Mol. Biol. Cell. 9 (1998) 3273-97.

[13] S.C. Stelpflug, R.S. Sekhon, B. Vaillancourt, C.N. Hirsch, C.R. Buell, N. de Leon, S.M. Kaeppler, An Expanded Maize Gene Expression Atlas based on RNA Sequencing and its Use to Explore Root Development, Plant Genome. 9 (2016) 314-362. doi:10.3835/plantgenome2015.04.0025.

[14] J.M. Stuart, E. Segal, D. Koller, S.K. Kim, A gene-coexpression network for global discovery of conserved genetic modules., Science. 302 (2003) 249-55. doi:10.1126/science.1087447.

[15] C.J. Wolfe, I.S. Kohane, A.J. Butte, Systematic survey reveals general applicability of "guilt-byassociation" within gene coexpression networks., BMC Bioinformatics. 6 (2005) 227. doi:10.1186/1471-2105-6-227.

[16] A. Brazma, J. Vilo, Gene expression data analysis., FEBS Lett. 480 (2000) 17-24.

[17] S. De Bodt, D. Carvajal, J. Hollunder, J. Van den Cruyce, S. Movahedi, D. Inzé, CORNET: a userfriendly tool for data mining and integration., Plant Physiol. 152 (2010) 1167-79. doi:10.1104/pp.109.147215.

[18] D. Steinhauser, B. Usadel, A. Luedemann, O. Thimm, J. Kopka, CSB.DB: a comprehensive systemsbiology database., Bioinformatics. 20 (2004) 3647-51. doi:10.1093/bioinformatics/bth398.

[19] C. Sánchez Claros, A. Tramontano, Detecting Mutually Exclusive Interactions in Protein-Protein Interaction Maps, PLoS One. 7 (2012) e38765. doi:10.1371/journal.pone.0038765.

[20] L. Song, P. Langfelder, S. Horvath, Comparison of co-expression measures: mutual information, correlation, and model based indices, BMC Bioinformatics. 13 (2012) 328. doi:10.1186/14712105-13-328.

[21] Y. Cheng, G. Church, Biclustering of expression data, Int. Conf. Intell. Syst. Mol. Biol. ISMB. Int. Conf. Intell. Syst. Mol. Biol. Dep. Genet. Harvard Med. Sch. Boston, MA 02115, USA. 8 (1999) 93103. doi:10.1007/11564126. 
[22] A. Prelić, S. Bleuler, P. Zimmermann, A. Wille, P. Bühlmann, W. Gruissem, L. Hennig, L. Thiele, E. Zitzler, A systematic comparison and evaluation of biclustering methods for gene expression data., Bioinformatics. 22 (2006) 1122-9. doi:10.1093/bioinformatics/btl060.

[23] J.D. Allen, Y. Xie, M. Chen, L. Girard, G. Xiao, Comparing statistical methods for constructing large scale gene networks., PLoS One. 7 (2012) e29348.

[24] S. Kumari, J. Nie, H.-S. Chen, H. Ma, R. Stewart, X. Li, M.-Z. Lu, W.M. Taylor, H. Wei, Evaluation of gene association methods for coexpression network construction and biological knowledge discovery., PLoS One. 7 (2012) e50411. doi:10.1371/journal.pone.0050411.

[25] K. Aoki, Y. Ogata, D. Shibata, Approaches for extracting practical information from gene coexpression networks in plant biology., Plant Cell Physiol. 48 (2007) 381-90. doi:10.1093/pcp/pcm013.

[26] B. Usadel, F. Poree, A. Nagel, M. Lohse, A. Czedik-Eysenberg, M. Stitt, A guide to using MapMan to visualize and compare Omics data in plants: a case study in the crop species, Maize., Plant. Cell Environ. 32 (2009) 1211-29. doi:10.1111/j.1365-3040.2009.01978.x.

[27] R. a. Fisher, R. a. Fisher, Frequency distribution of the values of the correlation coefficient in samples from an indefinitely large population, Biometrika. 10 (1915) 507-521. doi:10.2307/2331838.

[28] C.S. Greene, A. Krishnan, A.K. Wong, E. Ricciotti, R. a Zelaya, D.S. Himmelstein, R. Zhang, B.M. Hartmann, E. Zaslavsky, S.C. Sealfon, D.I. Chasman, G. a FitzGerald, K. Dolinski, T. Grosser, O.G. Troyanskaya, Understanding multicellular function and disease with human tissue-specific networks, Nat. Genet. 47 (2015) 569-576. doi:10.1038/ng.3259.

[29] C. Huttenhower, M. Schroeder, M.D. Chikina, O.G. Troyanskaya, The Sleipnir library for computational functional genomics., Bioinformatics. 24 (2008) 1559-61. doi:10.1093/bioinformatics/btn237.

[30] R. Swanson-Wagner, R. Briskine, R. Schaefer, M.B. Hufford, J. Ross-Ibarra, C.L. Myers, P. Tiffin, N.M. Springer, Reshaping of the maize transcriptome by domestication., Proc. Natl. Acad. Sci. U. S. A. 109 (2012) 11878-83. doi:10.1073/pnas.1201961109.

[31] F.N. David, The moments of the $z$ and F distributions., Biometrika. 36 (1949) 394-403. doi:10.1093/biomet/36.3-4.394.

[32] P. Langfelder, S. Horvath, WGCNA: an R package for weighted correlation network analysis., BMC Bioinformatics. 9 (2008) 559. doi:10.1186/1471-2105-9-559.

[33] A.J. Severin, J.L. Woody, Y.-T. Bolon, B. Joseph, B.W. Diers, A.D. Farmer, G.J. Muehlbauer, R.T. Nelson, D. Grant, J.E. Specht, M.A. Graham, S.B. Cannon, G.D. May, C.P. Vance, R.C. Shoemaker, RNA-Seq Atlas of Glycine max: a guide to the soybean transcriptome., BMC Plant Biol. 10 (2010) 160. doi:10.1186/1471-2229-10-160.

[34] A.-L. Barabási, Z.N. Oltvai, Network biology: understanding the cell's functional organization., Nat. Rev. Genet. 5 (2004) 101-113. doi:10.1038/nrg1272.

[35] J. Alstott, E. Bullmore, D. Plenz, powerlaw: a Python package for analysis of heavy-tailed distributions., PLoS One. 9 (2014) e85777. doi:10.1371/journal.pone.0085777. 
[36] R. Khanin, E. Wit, How Scale-Free Are Biological Networks, J. Comput. Biol. 13 (2006) 810-818. doi:10.1089/cmb.2006.13.810.

[37] G. Lima-Mendez, J. van Helden, The powerful law of the power law and other myths in network biology, Mol. Biosyst. 5 (2009) 1482. doi:10.1039/b908681a.

[38] K. Saito, M.Y. Hirai, K. Yonekura-Sakakibara, Decoding genes with coexpression networks and metabolomics - "majority report by precogs"., Trends Plant Sci. 13 (2008) 36-43.

doi:10.1016/j.tplants.2007.10.006.

[39] M. a Harris, J. Clark, a Ireland, J. Lomax, M. Ashburner, R. Foulger, K. Eilbeck, S. Lewis, B. Marshall, C. Mungall, J. Richter, G.M. Rubin, J. a Blake, C. Bult, M. Dolan, H. Drabkin, J.T. Eppig, D.P. Hill, L. Ni, M. Ringwald, R. Balakrishnan, J.M. Cherry, K.R. Christie, M.C. Costanzo, S.S. Dwight, S. Engel, D.G. Fisk, J.E. Hirschman, E.L. Hong, R.S. Nash, a Sethuraman, C.L. Theesfeld, D. Botstein, K. Dolinski, B. Feierbach, T. Berardini, S. Mundodi, S.Y. Rhee, R. Apweiler, D. Barrell, E. Camon, E. Dimmer, V. Lee, R. Chisholm, P. Gaudet, W. Kibbe, R. Kishore, E.M. Schwarz, P. Sternberg, M. Gwinn, L. Hannick, J. Wortman, M. Berriman, V. Wood, N. de la Cruz, P. Tonellato, P. Jaiswal, T. Seigfried, R. White, The Gene Ontology (GO) database and informatics resource., Nucleic Acids Res. 32 (2004) D258-61. doi:10.1093/nar/gkh036.

[40] M. Kanehisa, Y. Sato, M. Kawashima, M. Furumichi, M. Tanabe, KEGG as a reference resource for gene and protein annotation, Nucleic Acids Res. 44 (2016) D457-D462. doi:10.1093/nar/gkv1070.

[41] O. Thimm, O. Bläsing, Y. Gibon, A. Nagel, S. Meyer, P. Krüger, J. Selbig, L.A. Müller, S.Y. Rhee, M. Stitt, MAPMAN: A user-driven tool to display genomics data sets onto diagrams of metabolic pathways and other biological processes, Plant J. 37 (2004) 914-939. doi:10.1111/j.1365313X.2004.02016.x.

[42] B. Usadel, T. Obayashi, M. Mutwil, F.M. Giorgi, G.W. Bassel, M. Tanimoto, A. Chow, D. Steinhauser, S. Persson, N.J. Provart, Co-expression tools for plant biology: opportunities for hypothesis generation and caveats., Plant. Cell Environ. 32 (2009) 1633-51. doi:10.1111/j.13653040.2009.02040.x.

[43] K. Mochida, Y. Uehara-Yamaguchi, T. Yoshida, T. Sakurai, K. Shinozaki, Global landscape of a coexpressed gene network in barley and its application to gene discovery in Triticeae crops., Plant Cell Physiol. 52 (2011) 785-803. doi:10.1093/pcp/pcr035.

[44] O. Atias, B. Chor, D. a Chamovitz, Large-scale analysis of Arabidopsis transcription reveals a basal co-regulation network., BMC Syst. Biol. 3 (2009) 86. doi:10.1186/1752-0509-3-86.

[45] D.A. Orlando, S.M. Brady, J.D. Koch, J.R. Dinneny, P.N. Benfey, Manipulating large-scale Arabidopsis microarray expression data: identifying dominant expression patterns and biological process enrichment., Methods Mol. Biol. 553 (2009) 57-77. doi:10.1007/978-1-60327-563-7_4.

[46] S. van Dongen, MCL: A Cluster Algoithm for Graphs, (2000).

[47] M. Li, J. Chen, J. Wang, B. Hu, G. Chen, Modifying the DPClus algorithm for identifying protein complexes based on new topological structures., BMC Bioinformatics. 9 (2008) 398. doi:10.1186/1471-2105-9-398.

[48] M. Schena, D. Shalon, R.W. Davis, P.O. Brown, Quantitative monitoring of gene expression patterns with a complementary DNA microarray., Science. 270 (1995) 467-70. 
[49] J. Kilian, D. Whitehead, J. Horak, D. Wanke, S. Weinl, O. Batistic, C. D’Angelo, E. Bornberg-Bauer, J. Kudla, K. Harter, The AtGenExpress global stress expression data set: protocols, evaluation and model data analysis of UV-B light, drought and cold stress responses., Plant J. 50 (2007) 347-63. doi:10.1111/j.1365-313X.2007.03052.x.

[50] J.M. Lee, Genomic Gene Clustering Analysis of Pathways in Eukaryotes, Genome Res. 13 (2003) 875-882. doi:10.1101/gr.737703.

[51] T. Obayashi, Y. Okamura, S. Ito, S. Tadaka, Y. Aoki, M. Shirota, K. Kinoshita, ATTED-II in 2014: Evaluation of gene coexpression in agriculturally important plants, Plant Cell Physiol. 55 (2014) e6-e6. doi:10.1093/pcp/pct178.

[52] N.K. Sarkar, Y.-K. Kim, A. Grover, Coexpression network analysis associated with call of rice seedlings for encountering heat stress., Plant Mol. Biol. 84 (2014) 125-43. doi:10.1007/s11103013-0123-3.

[53] Z.-L. Zheng, Y. Zhao, Transcriptome comparison and gene coexpression network analysis provide a systems view of citrus response to "Candidatus Liberibacter asiaticus" infection., BMC Genomics. 14 (2013) 27. doi:10.1186/1471-2164-14-27.

[54] S.M. Brady, D.A. Orlando, J.-Y. Lee, J.Y. Wang, J. Koch, J.R. Dinneny, D. Mace, U. Ohler, P.N. Benfey, A high-resolution root spatiotemporal map reveals dominant expression patterns., Science. 318 (2007) 801-6. doi:10.1126/science.1146265.

[55] K.L. Childs, R.M. Davidson, C.R. Buell, Gene coexpression network analysis as a source of functional annotation for rice genes., PLoS One. 6 (2011) e22196.

doi:10.1371/journal.pone.0022196.

[56] F.-F. Fu, H.-W. Xue, Coexpression analysis identifies Rice Starch Regulator1, a rice AP2/EREBP family transcription factor, as a novel rice starch biosynthesis regulator., Plant Physiol. 154 (2010) 927-38. doi:10.1104/pp.110.159517.

[57] R.S. Sekhon, R. Briskine, C.N. Hirsch, C.L. Myers, N.M. Springer, C.R. Buell, N. de Leon, S.M. Kaeppler, Maize gene atlas developed by RNA sequencing and comparative evaluation of transcriptomes based on RNA sequencing and microarrays., PLoS One. 8 (2013) e61005. doi:10.1371/journal.pone.0061005.

[58] S. Ozaki, Y. Ogata, K. Suda, A. Kurabayashi, T. Suzuki, N. Yamamoto, Y. lijima, T. Tsugane, T. Fujii, C. Konishi, S. Inai, S. Bunsupa, M. Yamazaki, D. Shibata, K. Aoki, Coexpression analysis of tomato genes and experimental verification of coordinated expression of genes found in a functionally enriched coexpression module., DNA Res. 17 (2010) 105-16. doi:10.1093/dnares/dsq002.

[59] M. Obertello, S. Shrivastava, M.S. Katari, G.M. Coruzzi, Cross-Species Network Analysis Uncovers Conserved Nitrogen-Regulated Network Modules in Rice., Plant Physiol. 168 (2015) 1830-43. doi:10.1104/pp.114.255877.

[60] C. Ruprecht, M. Mutwil, F. Saxe, M. Eder, Z. Nikoloski, S. Persson, Large-Scale Co-Expression Approach to Dissect Secondary Cell Wall Formation Across Plant Species, Front. Plant Sci. 2 (2011) 1-13. doi:10.3389/fpls.2011.00023.

[61] K. Righetti, J.L. Vu, S. Pelletier, B.L. Vu, E. Glaab, D. Lalanne, A. Pasha, R. V Patel, N.J. Provart, J. Verdier, O. Leprince, J. Buitink, Inference of Longevity-Related Genes from a Robust Coexpression Network of Seed Maturation Identifies Regulators Linking Seed Storability to Biotic Defense- 
Related Pathways., Plant Cell. (2015) tpc.15.00632-. doi:10.1105/tpc.15.00632.

[62] L.G. Leal, C. López, L. López-Kleine, Construction and comparison of gene co-expression networks shows complex plant immune responses., PeerJ. 2 (2014) e610. doi:10.7717/peerj.610.

[63] M. Itkin, U. Heinig, O. Tzfadia, A.J. Bhide, B. Shinde, P.D. Cardenas, S.E. Bocobza, T. Unger, S. Malitsky, R. Finkers, Y. Tikunov, A. Bovy, Y. Chikate, P. Singh, I. Rogachev, J. Beekwilder, A.P. Giri, A. Aharoni, Biosynthesis of Antinutritional Alkaloids in Solanaceous Crops Is Mediated by Clustered Genes, Science (80-. ). 341 (2013) 175-179. doi:10.1126/science.1240230.

[64] C. Ruprecht, A. Mendrinna, T. Tohge, A. Sampathkumar, S. Klie, A.R. Fernie, Z. Nikoloski, S. Persson, M. Mutwil, FamNet: A framework to identify multiplied modules driving pathway diversification in plants., Plant Physiol. 170 (2016) pp.15.01281-. doi:10.1104/pp.15.01281.

[65] K. Cho, K. Cho, H. Sohn, I.J. Ha, S. Hong, Network analysis of the metabolome and transcriptome reveals novel regulation of potato pigmentation, J. Exp. Bot. 67 (2016) 1519-1533. doi:10.1093/jxb/erv549.

[66] J. Quackenbush, Microarray data normalization and transformation., Nat. Genet. 32 Suppl (2002) 496-501. doi:10.1038/ng1032.

[67] Z. Wang, M. Gerstein, M. Snyder, RNA-Seq: a revolutionary tool for transcriptomics., Nat. Rev. Genet. 10 (2009) 57-63. doi:10.1038/nrg2484.

[68] X. Tao, Y.-H. Gu, H.-Y. Wang, W. Zheng, X. Li, C.-W. Zhao, Y.-Z. Zhang, Digital Gene Expression Analysis Based on Integrated De Novo Transcriptome Assembly of Sweet Potato [Ipomoea batatas (L.) Lam.], PLoS One. 7 (2012) e36234. doi:10.1371/journal.pone.0036234.

[69] B.J. Haas, A. Papanicolaou, M. Yassour, M. Grabherr, P.D. Blood, J. Bowden, M.B. Couger, D. Eccles, B. Li, M. Lieber, M.D. Macmanes, M. Ott, J. Orvis, N. Pochet, F. Strozzi, N. Weeks, R. Westerman, T. William, C.N. Dewey, R. Henschel, R.D. Leduc, N. Friedman, A. Regev, De novo transcript sequence reconstruction from RNA-seq using the Trinity platform for reference generation and analysis., Nat. Protoc. 8 (2013) 1494-512. doi:10.1038/nprot.2013.084.

[70] M.H. Schulz, D.R. Zerbino, M. Vingron, E. Birney, Oases: robust de novo RNA-seq assembly across the dynamic range of expression levels., Bioinformatics. 28 (2012) 1086-92. doi:10.1093/bioinformatics/bts094.

[71] A. Rafalski, M. Morgante, Corn and humans: Recombination and linkage disequilibrium in two genomes of similar size, Trends Genet. 20 (2004) 103-111. doi:10.1016/j.tig.2003.12.002.

[72] J.-M. Chia, C. Song, P.J. Bradbury, D. Costich, N. de Leon, J. Doebley, R.J. Elshire, B. Gaut, L. Geller, J.C. Glaubitz, M. Gore, K.E. Guill, J. Holland, M.B. Hufford, J. Lai, M. Li, X. Liu, Y. Lu, R. McCombie, R. Nelson, J. Poland, B.M. Prasanna, T. Pyhäjärvi, T. Rong, R.S. Sekhon, Q. Sun, M.I. Tenaillon, F. Tian, J. Wang, X. Xu, Z. Zhang, S.M. Kaeppler, J. Ross-Ibarra, M.D. McMullen, E.S. Buckler, G. Zhang, Y. Xu, D. Ware, Maize HapMap2 identifies extant variation from a genome in flux., Nat. Genet. 44 (2012) 803-7. doi:10.1038/ng.2313.

[73] C.N. Hirsch, J.M. Foerster, J.M. Johnson, R.S. Sekhon, G. Muttoni, B. Vaillancourt, F. Peñagaricano, E. Lindquist, M.A. Pedraza, K. Barry, N. de Leon, S.M. Kaeppler, C.R. Buell, Insights into the maize pan-genome and pan-transcriptome., Plant Cell. 26 (2014) 121-35. doi:10.1105/tpc.113.119982.

[74] N.M. Springer, K. Ying, Y. Fu, T. Ji, C.-T. Yeh, Y. Jia, W. Wu, T. Richmond, J. Kitzman, H. 
Rosenbaum, A.L. Iniguez, W.B. Barbazuk, J.A. Jeddeloh, D. Nettleton, P.S. Schnable, Maize inbreds exhibit high levels of copy number variation (CNV) and presence/absence variation (PAV) in genome content., PLoS Genet. 5 (2009) e1000734. doi:10.1371/journal.pgen.1000734.

[75] J. Lai, R. Li, X. Xu, W. Jin, M. Xu, H. Zhao, Z. Xiang, W. Song, K. Ying, M. Zhang, Y. Jiao, P. Ni, J. Zhang, D. Li, X. Guo, K. Ye, M. Jian, B. Wang, H. Zheng, H. Liang, X. Zhang, S. Wang, S. Chen, J. Li, Y. Fu, N.M. Springer, H. Yang, J.J. Wang, J. Dai, P.S. Schnable, J.J. Wang, Genome-wide patterns of genetic variation among elite maize inbred lines., Nat. Genet. 42 (2010) 1027-30. doi:10.1038/ng.684.

[76] R.A. Swanson-Wagner, S.R. Eichten, S. Kumari, P. Tiffin, J.C. Stein, D. Ware, N.M. Springer, Pervasive gene content variation and copy number variation in maize and its undomesticated progenitor., Genome Res. 20 (2010) 1689-99. doi:10.1101/gr.109165.110.

[77] S.P. Ficklin, F. Luo, F.A. Feltus, The association of multiple interacting genes with specific phenotypes in rice using gene coexpression networks., Plant Physiol. 154 (2010) 13-24. doi:10.1104/pp.110.159459.

[78] T. Zhu, P. Budworth, W. Chen, N. Provart, H.-S. Chang, S. Guimil, W. Su, B. Estes, G. Zou, X. Wang, Transcriptional control of nutrient partitioning during rice grain filling., Plant Biotechnol. J. 1 (2003) 59-70. doi:10.1046/j.1467-7652.2003.00006.x.

[79] M.D. McMullen, S. Kresovich, H.S. Villeda, P. Bradbury, H. Li, Q. Sun, S. Flint-Garcia, J. Thornsberry, C. Acharya, C. Bottoms, P. Brown, C. Browne, M. Eller, K. Guill, C. Harjes, D. Kroon, N. Lepak, S.E. Mitchell, B. Peterson, G. Pressoir, S. Romero, M. Oropeza Rosas, S. Salvo, H. Yates, M. Hanson, E. Jones, S. Smith, J.C. Glaubitz, M. Goodman, D. Ware, J.B. Holland, E.S. Buckler, Genetic properties of the maize nested association mapping population., Science. 325 (2009) 737-40. doi:10.1126/science.1174320.

[80] M. Nordborg, J.O. Borevitz, J. Bergelson, C.C. Berry, J. Chory, J. Hagenblad, M. Kreitman, J.N. Maloof, T. Noyes, P.J. Oefner, E.A. Stahl, D. Weigel, The extent of linkage disequilibrium in Arabidopsis thaliana., Nat. Genet. 30 (2002) 190-3. doi:10.1038/ng813.

[81] K.S. Caldwell, J. Russell, P. Langridge, W. Powell, Extreme population-dependent linkage disequilibrium detected in an inbreeding plant species, Hordeum vulgare, Genetics. 172 (2006) 557-567. doi:10.1534/genetics.104.038489.

[82] P.L. Morrell, D.M. Toleno, K.E. Lundy, M.T. Clegg, Low levels of linkage disequilibrium in wild barley (Hordeum vulgare ssp. spontaneum) despite high rates of self-fertilization., Proc. Natl. Acad. Sci. U. S. A. 102 (2005) 2442-2447. doi:10.1073/pnas.0409804102.

[83] J.G. Wallace, P.J. Bradbury, N. Zhang, Y. Gibon, M. Stitt, E.S. Buckler, Association mapping across numerous traits reveals patterns of functional variation in maize., PLoS Genet. 10 (2014) e1004845. doi:10.1371/journal.pgen.1004845.

[84] X. Li, C. Zhu, C.-T. Yeh, W. Wu, E.M. Takacs, K.A. Petsch, F. Tian, G. Bai, E.S. Buckler, G.J. Muehlbauer, M.C.P. Timmermans, M.J. Scanlon, P.S. Schnable, J. Yu, Genic and nongenic contributions to natural variation of quantitative traits in maize., Genome Res. 22 (2012) 243644. doi:10.1101/gr.140277.112.

[85] F. Tian, P.J. Bradbury, P.J. Brown, H. Hung, Q. Sun, S. Flint-Garcia, T.R. Rocheford, M.D. McMullen, J.B. Holland, E.S. Buckler, Genome-wide association study of leaf architecture in the maize nested 
association mapping population., Nat. Genet. 43 (2011) 159-62. doi:10.1038/ng.746.

[86] A. Druka, G. Muehlbauer, I. Druka, R. Caldo, U. Baumann, N. Rostoks, A. Schreiber, R. Wise, T. Close, A. Kleinhofs, A. Graner, A. Schulman, P. Langridge, K. Sato, P. Hayes, J. McNicol, D. Marshall, R. Waugh, An atlas of gene expression from seed to seed through barley development., Funct. Integr. Genomics. 6 (2006) 202-11. doi:10.1007/s10142-006-0025-4.

[87] M. Nakazono, F. Qiu, L.A. Borsuk, P.S. Schnable, Laser-capture microdissection, a tool for the global analysis of gene expression in specific plant cell types: identification of genes expressed differentially in epidermal cells or vascular tissues of maize., Plant Cell. 15 (2003) 583-96. doi:10.1105/tpc.008102. However.

[88] E.M. Takacs, J. Li, C. Du, L. Ponnala, D. Janick-Buckner, J. Yu, G.J. Muehlbauer, P.S. Schnable, M.C.P. Timmermans, Q. Sun, D. Nettleton, M.J. Scanlon, Ontogeny of the Maize Shoot Apical Meristem, Plant Cell. 24 (2012) 3219-3234. doi:10.1105/tpc.112.099614.

[89] J. Zhan, D. Thakare, C. Ma, A. Lloyd, N.M. Nixon, A.M. Arakaki, W.J. Burnett, K.O. Logan, D. Wang, X. Wang, G.N. Drews, R. Yadegari, RNA sequencing of laser-capture microdissected compartments of the maize kernel identifies regulatory modules associated with endosperm cell differentiation, Plant Cell. 27 (2015) 513-531. doi:10.1105/tpc.114.135657.

[90] M. Shen, C.D. Broeckling, E.Y. Chu, G. Ziegler, I.R. Baxter, J.E. Prenni, O. a. Hoekenga, Leveraging Non-Targeted Metabolite Profiling via Statistical Genomics, PLoS One. 8 (2013). doi:10.1371/journal.pone.0057667.

[91] M. Civelek, A.J. Lusis, Systems genetics approaches to understand complex traits., Nat. Rev. Genet. 15 (2014) 34-48. doi:10.1038/nrg3575.

[92] D.J. Weston, L.E. Gunter, A. Rogers, S.D. Wullschleger, Connecting genes, coexpression modules, and molecular signatures to environmental stress phenotypes in plants., BMC Syst. Biol. 2 (2008) 16. doi:10.1186/1752-0509-2-16.

[93] M. Filteau, S.A. Pavey, J. St-Cyr, L. Bernatchez, Gene coexpression networks reveal key drivers of phenotypic divergence in lake whitefish, Mol. Biol. Evol. 30 (2013) 1384-1396. doi:10.1093/molbev/mst053.

[94] J.P. Gallagher, C.E. Grover, G. Hu, J.F. Wendel, Insights into the Ecology and Evolution of Polyploid Plants through Network Analysis, Mol. Ecol. (2016) n/a-n/a. doi:10.1111/mec.13626.

[95] G.C. Conant, K.H. Wolfe, Functional partitioning of yeast co-expression networks after genome duplication, PLoS Biol. 4 (2006) 545-554. doi:10.1371/journal.pbio.0040109.

[96] C.H. Waddington, Torwards a Theoretical Biology, Nature. 218 (1968) 639-640. 